\title{
ALOFÁN, UNA NANOPARTÍCULA NATURAL PRESENTE EN ANDISOLES DEL ECUADOR, PROPIEDADES Y APLICACIONES
}

\author{
ALLOPHANE, A NATURAL NANOPARTICLE PRESENT IN \\ ANDISOLES OF ECUADOR, PROPERTIES AND APPLICATIONS
}

\author{
Jorge Silva-Yumi ${ }^{1,2,3 *}{ }^{\mathbb{0}}$, Roberto Cazorla Martínez ${ }^{4}{ }^{\oplus}$, Carlos Medina \\ Serrano $^{1,3} 3_{(0)}$ y Gabriela Chango Lescano ${ }^{2}$ (i)
}

\author{
${ }^{1}$ Facultad de Ciencias, Escuela Superior Politécnica de Chimborazo, EC060155, Riobamba, Ecuador. \\ ${ }^{2}$ Grupo de Investigación y Desarrollo Tecnológico de Energías Renovables (GIDETER), Facultad de Mecánica, Escuela Superior \\ Politécnica de Chimborazo, EC060155, Riobamba, Ecuador. \\ ${ }^{3}$ Grupo de Investigación en Materiales Avanzados (GIMA), Facultad de Ciencias, Escuela Superior Politécnica de Chimborazo, \\ EC060155, Riobamba, Ecuador. \\ ${ }^{4}$ Ministerio de Agricultura y Ganadería, Dirección Distrital 06 D01 Chambo, EC060155, Chambo, Ecuador. \\ *Autor para correspondencia: jorge.silvay@espoch.edu.ec
}

\section{Resumen}

El alofán es una nanopartícula natural presente en suelos de origen volcánico como los andisoles, que se encuentran distribuidos alrededor de todo el mundo en países con actividad volcánica. En Ecuador, los andisoles constituyen el $30 \%$ del territorio en zonas altas y húmedas de la región sierra-norte. El alofán se puede obtener de los andisoles a través de procesos físicos y químicos, o a su vez se puede sintetizar. Este nanomaterial posee múltiples propiedades para varias aplicaciones en diferentes áreas. Existen muchas investigaciones de estas nanopartículas y de este tipo de suelos, pero no se han estudiado aún en el Ecuador. En este artículo se presentan las características estructurales, propiedades, la formación, aislamiento, síntesis y usos del alofán, con el fin de generar conocimiento e incentivar la investigación de estos suelos que son fuente de la mencionada nanopartícula. La búsqueda de literatura se realizó en bases de datos de Science Direct y Google Académico, y se utilizaron artículos de alto impacto relacionados con investigaciones de alofán natural o sintético. Las características particulares que tiene el alofán le permite ser usado como remediador ambiental, bactericida, antiinflamatorio, ignífugo, soporte de enzimas, pero además se ha estudiado en catálisis, fotocatálisis y electrocatálisis. Al considerar el área cubierta por el alofán en el territorio ecuatoriano y su disponibilidad, este se puede aprovechar para realizar investigaciones basadas en los estudios internacionales que se han desarrollado para aprovechar en el área ambiental y médica.
\end{abstract}

Palabras clave: nanopartícula natural, andisol, suelos de origen volcánico, halloysita, imogolita. 


\begin{abstract}
The allophane is a natural nanoparticle present in soils from volcanic origin such as andisols, which are distributed worldwide, especially in countries that have active volcanoes. In Ecuador, andisols are in high and humid areas from the Highland/North region, constituting $30 \%$ of the territory. The allophane can be obtained from andisols through physical and chemical processes or it can be also synthesized. This nanomaterial has multiple properties for various applications in different areas; and there are studies about these nanoparticles and this kind of soil, but they have not yet been conducted in Ecuador. This article presents a review of structural characteristics, properties, formation, isolation, synthesis and uses of allophane to extend knowledge and encourage the conduction of research in these soils, which are the source of the aforementioned nanoparticle. The literature review was performed on Science Direct and Google Scholar databases using high impact articles related to natural or synthetic allophane. Allophane has characteristics that allow it to be used as an environmental remediator, bactericidal, anti-inflammatory, flame retardant, enzyme support and also in catalysis, photocatalysis and electrocatalysis. Considering the availability and the large area covered by andisols in Ecuador, research based on international investigations can be perform to take advantage of it.
\end{abstract}

Keywords: natural nanoparticle, andosol, volcanic soils, halloysite, imogolite.

Forma sugerida de citar: Silva-Yumi, J., Cazorla-Martínez, R., Medina-Serrano, C. y Chango Lescano, G. (2021). Alofán, una nanopartícula natural presente en andisoles del Ecuador, propiedades y aplicaciones. La Granja: Revista de Ciencias de la Vida. Vol. 33(1):53-66. http://doi. org/10.17163/lgr.n33.2021.05.

IDs Orcid:

Jorge Silva Yumi: http:/ / orcid.org/0000-0002-6005-9915

Roberto Cazorla Martínez: http:/ /orcid.org/0000-0001-9752-3577

Carlos Medina Serrano: http:/ /orcid.org/0000-0003-4916-7242

Gabriela Chango Lescano: http://orcid.org/0000-0003-0228-7095 


\section{Introducción}

El alofán es una nanopartícula natural (Nishikiori y col., 2012) presente en suelos de origen volcánico, denominados andosoles según la base referencial mundial del recurso suelo (Vistoso y col., 2012) o andisoles según Soil Taxonomy NRCS/USDA. Los andosoles o andisoles son suelos que se forman a partir de materiales volcánicos como cenizas, a través de procesos de meteorización bajo condiciones ácidas en climas húmedos (Cervini-Silva y col., 2015; Saeki, Sakai y Wada, 2010), y se encuentran distribuidos alrededor de todo el mundo en regiones con una considerable actividad volcánica.

La partícula de alofán tiene la forma de una esfera porosa, con un diámetro entre 3 a $5 \mathrm{~nm}$ (Henmi y Wada, 1976), y está estructurada por una capa externa constituida por octaedros de aluminio (Al) y una capa interna constituida por tetraedros de silicio (Si). Presenta una gran área superficial, alta porosidad y carga variable, propiedades que permiten potenciales aplicaciones como adsorbente de cationes (Silva-Yumi y col., 2018), aniones (Nishikiori y col., 2017), compuestos derivados del benceno, ácidos grasos, detergentes, compuestos organoclorados (Arakawa, Matsuura y Okamoto, 2014; Baldermann y col., 2018; Garrido-Ramírez y col., 2013), ADN y aminoácidos (Saeki, Sakai y Wada, 2010), enzimas (Yu-Huang y col., 2016), en el área de catálisis (Garrido-Ramírez y col., 2013), en la elaboración de electrodos (Nishikiori y col., 2014), como ignífugo (Iyoda y col., 2012; Shukla y col., 2013) como elemento de tinta para impresoras (Arakawa, Matsuura y Okamoto, 2014), como anti inflamatorio y bactericida (Calabi-Floody y col., 2012), para la purificación de biodiesel (Yu-Huang y col., 2016), etc.

En Ecuador los andisoles constituyen alrededor del 30\% del área territorial, y se encuentran distribuidos en la región sierra centro-norte en las zonas altas y húmedas, aunque se extienden hacia la costa y el oriente (Calvache, 2014). Las investigaciones realizadas en torno a suelos de origen volcánico ecuatorianos se han enfocado en la pedogénesis (Zehetner, Miller y West, 2003), la estabilización y almacenamiento de carbono (C), los efectos del sobrepastoreo sobre la vegetación (Podwojewski y col., 2002), los efectos a corto plazo del manejo en la estructura del suelo de origen volcánico endureci- do en las capas profundas, la variabilidad de suelos de origen volcánico y su relación con el material parental, clima y su uso (Podwojewski y Germain, 2005), la meteorización química (Poncelet y Jouhannaud, 2013) y la presencia de n-metil cetonas como productos de la degradación de n-alcanos (Jansen y Nierop, 2009); sin embargo, ninguno de estos artículos menciona o se ha enfocado en el estudio de las nanopartículas de alofán presentes en este tipo de suelos, solo un artículo publicado en el 2007 estudia la influencia del contenido de alofán y la materia orgánica en las propiedades de los suelos de origen volcánico (Buytaert, Deckers y Wyseure, 2007).

En el año 2009 se publicó un artículo acerca de la existencia de un depósito masivo de alofán ubicado en la comunidad de San José de Achotillo, en la provincia de Santo Domingo de los Tsáchilas, su cuantificación se realizó en el año 2010 y se encontró la presencia de un alto contenido de alofán $(>60 \%)$, así como de halloysita que es otra nanopartícula natural de características similares; la presencia de óxidos de hierro y un bajo contenido de materia orgánica (Kaufhold y col., 2010). A partir de este año han surgido varias investigaciones en torno al alofán presente en este yacimiento, enfocándose en la adsorción de aniones como fluoruro y su comparación con otros adsorbentes similares (Kaufhold y col., 2010), la adsorción de cationes: bario, cobalto, estroncio y zinc (Baldermann y col., 2018), la actividad antiinflamatoria del alofán presente en este yacimiento (Cervini-Silva y col., 2015), así como su citotoxicidad (Cervini-Silva y col., 2014), y la activación para ser utilizado como catalizador (Vaca y Lalangui, 2018).

Los estudios sobre los andisoles ecuatorianos y las nanopartículas de alofán son limitados, y las investigaciones sobre alofán se han realizado desde instituciones ubicadas fuera del país como se observa en las filiaciones de los autores de las publicaciones. Si bien la producción científica de Ecuador se ha incrementado en los últimos años (Araujo-Bilmonte, Huertas-Tulcanaza y PárragaStead, 2020), se cuenta con personal capacitado que ha retornado desde el exterior para realizar investigación en esta área y con instituciones de educación superior con capacidad para desarrollar investigación en nanociencia y nanotecnología (Gutiérrez Coronado, 2018). Tal vez una de las limitantes es el 
desconocimiento de la existencia de este nanomaterial, razón por la cual el objetivo de este artículo es dar a conocer las características, propiedades, formación, aislamiento, síntesis y usos del alofán, con el fin de resaltar la potencialidad que puede tener este tipo de suelos que cubre una gran área del territorio ecuatoriano.

\section{Metodología}

La búsqueda y recopilación de los artículos utilizados en la revisión se efectuó entre enero de 2018 y enero de 2019 a través de las bases de datos: Scopus, Sciencedirect y Google Académico. Los criterios fueron los siguientes: (1) palabras clave: allophane, alofán, andisol Ecuador, andosol Ecuador, allophane Ecuador, alofán Ecuador, con algoritmos booleanos and y with. (2) tipo de artículos: investigación y revisión, (3) criterios de inclusión y exclusión: artículos enfocados en el estudio de alofán, artículos en cuartil 1 según Scimago Journal Country Rank para para los documentos en inglés, (4) documentos publicados dentro de los 10 últimos años.

Se han considerado 59 documentos entre artículos y trabajos de congresos, dos documentos de referencia y una patente, de los cuales se han extraído las principales ideas que se comparten en este documento. Los artículos han sido considerados en función de su información acerca del origen, síntesis, propiedades y aplicaciones de alofán.

\section{Andisoles en el mundo y Ecuador}

Los andisoles son suelos que se forman a partir de materiales volcánicos como cenizas, a través de procesos de meteorización bajo condiciones ácidas en climas húmedos (FAO, 2014; FAO, 2015), y se encuentran distribuidos alrededor de todo el mundo en regiones con una considerable actividad volcánica. En Asia y el pacífico se encuentran en Japón, Corea, Filipinas, Indonesia, Papúa Nueva Guinea y Nueva Zelanda. En Europa se hallan en Italia y Francia; en África y Oceanía se encuentran en Kenia, Ruanda, Tanzania, Etiopía y las Islas Canarias. En América se encuentran en países ubicados alrededor del cinturón de fuego del pacífico: Alaska, Estados Unidos, México (Pérez y col., 2016), Guatemala, El Salvador, Honduras, Nicaragua, Costa Rica (Alvarado, Mata y Chinchilla, 2014), Panamá,
Las Antillas, Colombia, Ecuador, Chile y Argentina.

En Ecuador si bien existen varios tipos de suelos, los andisoles constituyen $60593 \mathrm{~km}^{2}$ del territorio ecuatoriano (Fig. 1), y se encuentran distribuidos alrededor de la región sierra centro-norte en las zonas altas y húmedas, aunque se extienden también hacia la costa y el oriente (Calvache, 2015; Gonzáles, 2015; González, 2010). Son el resultado de la meteorización del material piroclástico producto de las erupciones de numerosos volcanes ubicados en la llamada "avenida de los volcanes", con más de 100 volcanes, cinco de ellos en actividad. Son suelos con una baja densidad aparente, por lo general con alto contenido de materia orgánica, baja resistencia al corte tangencial, buen drenaje, pero a su vez, con una buena retención de humedad (Calvache, 2015; FAO, 2015; Gonzáles, 2015).

\section{Formación}

El alofán se forma por la rápida meteorización del vidrio volcánico, cuando el $\mathrm{pH}$ del suelo es 5, el silicio y el aluminio se liberan desde el material volcánico reaccionando y formando alofán además de imogolita y halloysita, otros de los minerales presentes en este tipo de suelos y que también pueden formarse a partir de la hidrólisis de minerales primarios. La formación y persistencia de alofán, imogolita o halloysita es influenciada por el contenido de materia orgánica y el régimen de precipitación. El alofán predomina en suelos sometidos a un régimen de alta precipitación, mientras que la halloysita sobresale en suelos con un régimen de baja precipitación. Estos minerales están presentes en horizontes del suelo con bajo contenido de materia orgánica en donde el aluminio no existe como complejos con ácidos orgánicos o sustancias húmicas sino en forma de complejos inorgánicos (Yuan y Wada, 2012).

El alofán se puede extraer a partir de suelos de origen volcánico a través de una serie de pasos que implican de manera general la eliminación de materia orgánica, eliminación de óxidos de hierro, y separación de las fracciones nanométricas de las fracciones de arena, limo y arcilla. Esta nanopartícula se puede también sintetizar por el método de co-precipitación o por el método sol-gel. El primer método utiliza como precursores ácido or- 
tosilícico $\left(\mathrm{H}_{4} \mathrm{SiO}_{4}\right)$ u ortosilicato de sodio $\left(\mathrm{Na}_{4} \mathrm{SiO}_{4}\right)$ y cloruro de aluminio $\left(\mathrm{AlCl}_{3}\right)$ o perclorato de aluminio $\left(\mathrm{Al}\left(\mathrm{ClO}_{4}\right)_{3}\right)$. El método sol-gel que implica una hidrólisis de los reactivos, y la condensación de los productos obtenidos utiliza tetraetíl ortosilicato (TEOS) $\left(\mathrm{Si}\left(\mathrm{OC}_{2} \mathrm{H}_{5}\right)_{4}\right)$ y cloruro de aluminio $\left(\mathrm{AlCl}_{3}\right)$.
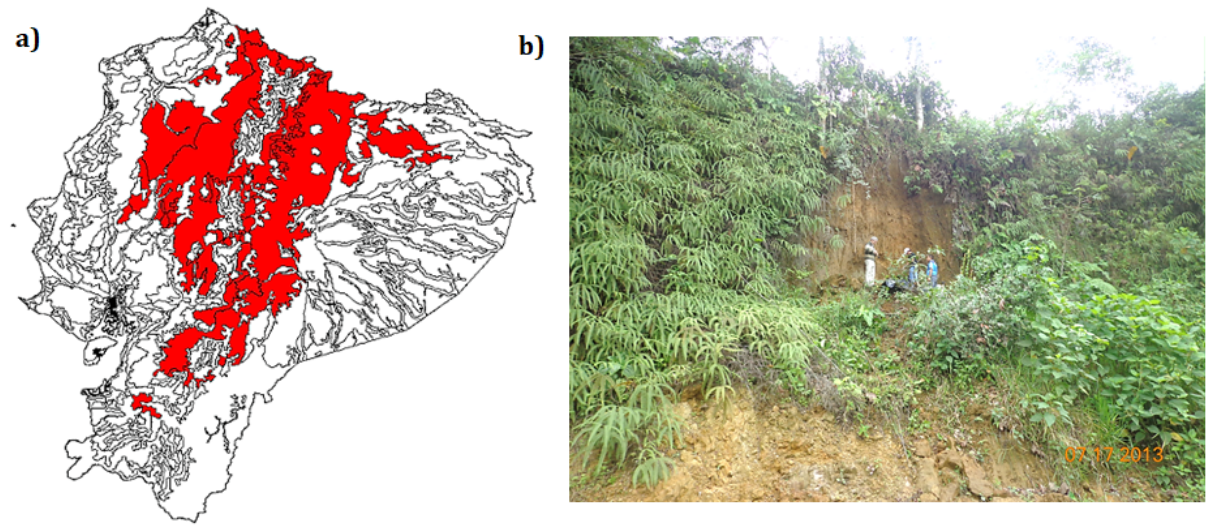

Figura 1. a) Área superficial del Ecuador cubierta por suelos de origen volcánico tomada de Calvache (2015). b) Yacimiento rico en alofán ubicado en Santo Domingo de los Tsáchilas (imagen propia).

\section{Características estructurales}

La partícula de alofán tiene la forma de una esfera porosa (Fig. 2) con diámetro externo entre 3,5 y 5,0 nm, radio interno entre $1,0-2,0 \mathrm{~nm}$ y una pared con un espesor entre 0,7 y $1,0 \mathrm{~nm}$. Posee poros con diámetro alrededor de 0,3-0,4 nm y presenta un área superficial específica determinada mediante el método EGME (etilenglicol monoetil eter) que oscila entre $400-900 \mathrm{~m}^{2} \mathrm{~g}^{-1}$. La pared externa está compuesta de octaedros de aluminio, mientras que la pared interna por tetraedros de silicio (Fig. 2), aunque es posible encontrar aluminio tanto en tetraedros como en octaedros.

La composición química del alofán cambia de acuerdo al predominio de $\mathrm{Al}$ o Si. En la naturaleza es posible encontrar alofán rico en aluminio $(A l / S i=2)$ y alofán rico en silicio $(A l / S i=1)$, por lo que su fórmula química se puede representar como $1-2 \mathrm{SiO}_{2} \cdot \mathrm{Al}_{2} \mathrm{O}_{3}, 5-6 \mathrm{H}_{2} \mathrm{O}$. La presencia de grupos silanol $(\mathrm{Si}-\mathrm{OH})$ en la superficie de la pared interna y grupos aluminiol $(\mathrm{Al}-\mathrm{OH})$ situados en la pared externa le permiten al alofán junto con la carga permanente o estructural que puede poseer, producto de la sustitución isomórfica de $A l^{3+}$ por $\mathrm{Si}^{4+}$, adquirir carga negativa o positiva (Fig. 3) en función de las condiciones del medio.

\section{Aplicaciones}

\subsection{Adsorbente para remediación ambien- tal}

La alta porosidad que posee el alofán, su gran área superficial y la carga que puede adoptar en función del medio hacen de él un potencial adsorbente tanto de especies con carga positiva (cationes), así como de aquellas con carga negativa (aniones), siendo los sitios internos (silanol) como externos (aluminol) (Reinert y col., 2011) los responsables del proceso. os procesos de adsorción pueden ser utilizados para el tratamiento de aguas de consumo y aguas residuales, ya que permiten que los contaminantes (especies orgánicas o inorgánicas), queden retenidos en la superficie del material y puedan ser removidos del medio acuoso.

Uno de los procesos que se ha estudiado con mayor frecuencia es la adsorción de fosfato debido a su particular fijación sobre el alofán, aunque también se ha estudiado la adsorción de molibdado (Elhadi y Henmi, 2000), arsenato, ácido silícico, ácido borico, cromato (Opiso, Sato y Yoneda, 2009), selenato, sulfato, oxalato, nitrato, ácido ortosilicico, fluoruro (Kaufhold y col., 2010), citrato, entre otras especies aniónicas. La adsorción de especies catiónicas ha sido menos estudiada en relación a las 
a)

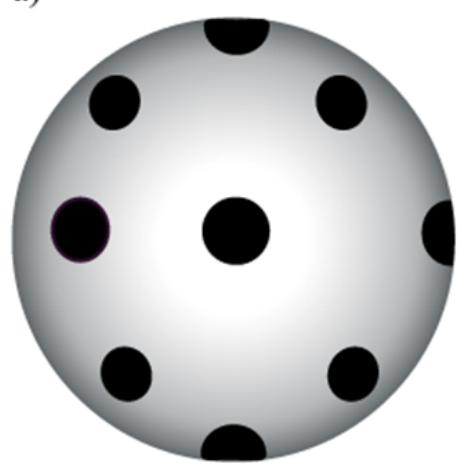

b)

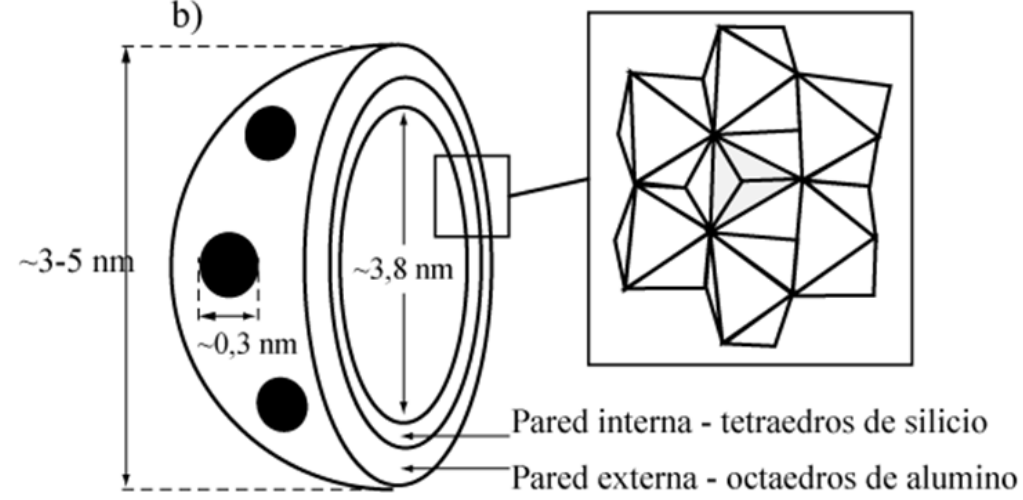

Figura 2. Morfología externa de una nanopartícula de alofán (a), detalle de la estructura interna (b).

especies aniónicas; entre las pocas especies estudiadas se puede mencionar cinc, cesio, cobre, cobalto, cadmio, bario y estroncio (Baldermann y col., 2018; Silva-Yumi y col., 2018).

La adsorción de especies orgánicas ha sido también de interés para los científicos, de la cuales se pueden citar 2,4-diclorofenol, pentaclorofenol, ácido húmico, benceno, ácido benzoico, ácido ftálico, benzaldehido, etil benzoato, dietil ftalato, ácido acético, ácido oxálico, ácido cítrico, polímeros como xantan, detergentes y ácidos grasos (Nishikiori2010). En la Figura 4 se pueden ver algunas de las estructuras que corresponden a las mencionadas especies y la manera cómo se adsorben sobre la superficie del alofán. La adsorción de gases se ha estudiado en mucho menor extensión, y se puede mencionar la adsorción de amoniaco (Zaenal, Matsue y Henmi, 2013).

\subsection{Adsorción de moléculas esenciales y origen de la vida}

Entender los procesos que ocurren dentro de este tipo de suelos ha sido posible gracias a estudios llevados a cabo, como por ejemplo los de Hashizume, Theng y Yamagishi (2002) y Hashizume y Theng (2007) en los que se ha determinado la alta afinidad del alofán por los nucleótidos, lo que implica su posible papel en la formación abiótica de polinucleóti- dos de tipo ARN, aunque a su vez la inmovilización de estos nucleótidos por complejación podría dificultar la oligomerización de ARN. La persistencia y supervivencia de aminoácidos en los suelos se ha atribuido a la adsorción y protección por arcillas y otros minerales. Por ejemplo, se ha observado que la adsorción de DL-alanina es mayor sobre alofán en suspensión y depende del pH. En la adsorción de D- y L-alanina y sus respectivos dímeros sobre alofán con distinta relación $\mathrm{Al} / \mathrm{Si}$ se aprecia una preferencia por L-alanil-L-alanina, sugiriendo que el tamaño, la separación de la carga intramolecular y la orientación superficial son los factores responsables.

Por otra parte, con el fin de reconstruir y estudiar ambientes pasados, así como las comunidades microbianas del suelo y el almacenamiento de carbono, la adsorción de ADN sobre el alofán ha sido estudiada por varios investigadores (Huang y Rawlence, 2014; Matsuura y col., 2013; Yu-Huang y col., 2016). Los resultados muestran que la adsorción de ADN es facilitada por la interacción de los grupos fosfato con los grupos $\mathrm{Al}-\mathrm{OH}$ del alofán, aunque es menor que la adsorción de adenosina- $5^{\prime}$ monofosfato (5'-AMP), molécula que se utiliza como referencia (Matsuura y col., 2013), razón por la cual se observa mayor afinidad por 5'-AMP que por adenina, adenosina o ribosa, debido también a la presencia de grupos fosfato que facilitan la interacción con los grupos $\mathrm{Al}-\mathrm{OH}$. 
La adsorción de ADN casi no se ve afectada por la fuerza iónica y disminuye al aumentar el $\mathrm{pH}$, la presencia de fosfato debido a la desprotonación de los grupos $\mathrm{Al}-\mathrm{OH}$ (Matsuura y col., 2013; Saeki, Sakai y Wada, 2010) producto de la competición por los sitios activos. La presencia de ácido húmico provoca también la disminución de adsorción debido a que ocupa los sitios activos del alofán (Yu-Huang y col., 2016). La adsorción de ADN es más alta so- bre el alofán que sobre sílice y montmorillonita pero más baja sobre gibbsita y goethita. La interacción de ADN y 5'-AMP con los grupos $(\mathrm{OH}) \mathrm{Al}\left(\mathrm{OH}_{2}\right)$ presentes en los ultramicroporos del alofán también se ha estudiado a través de simulación por computadora, y ha mostrado que el ADN sufre elongación y que la cadena principal de fosfato se altera después de unirse al alofán (Matsuura, Arakawa y Okamoto, 2014).

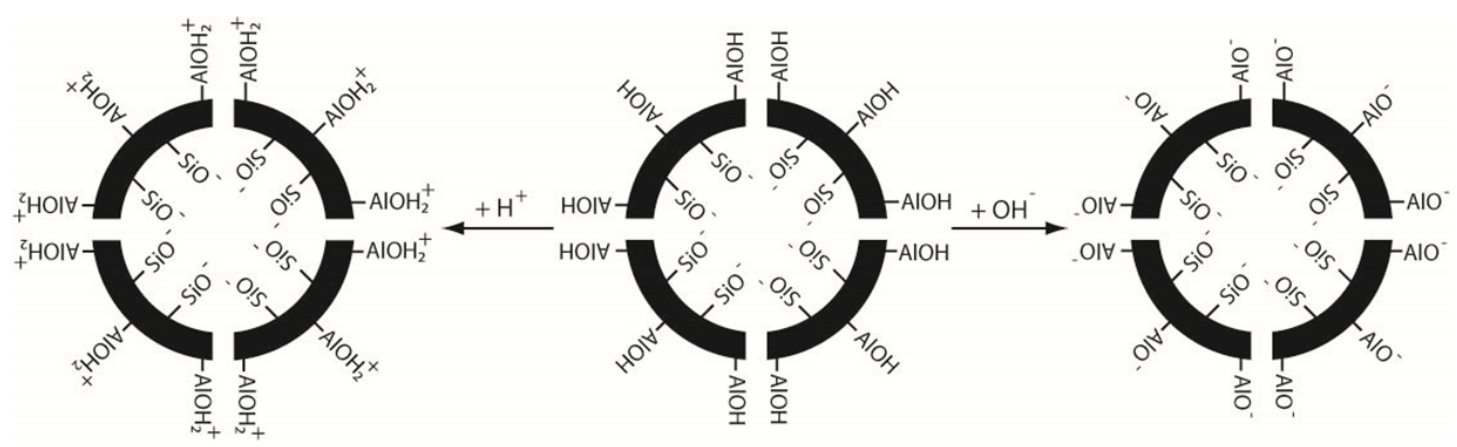

Figura 3. Carga superficial de la partícula de alofán en función del pH.

\subsection{Secuestro, estabilización de carbono y control del efecto invernadero}

$\mathrm{Al}$ ser los suelos el mayor reservorio de carbono y materia orgánica, es necesario el estudio de su dinámica, así como de las interacciones físico-químicas con los principales minerales presentes en estos, debido al papel como un potencial almacenador de $\mathrm{CO}_{2}$, y por tanto clave para el control del efecto invernadero (Huang y Rawlence, 2014; Triomphe y Livermore, 2005), así como para la protección contra la mineralización y la degradación microbiana.

La estabilización del carbono en suelos alofánicos es conflictiva, ya que por un lado es atribuida al $A l^{3+}$ y sesquioxidos, y por otro también se ha establecido una relación entre el carbono y el contenido de alofán (Garrido-Ramírez y col., 2012), observándose que la cantidad de materia orgánica enlazada es mayor sobre el material tipo imogolita (proto imogolita, proto imogolita tipo alofán) que sobre gibbsita o feldespatos no meteorizados. Esto demuestra que los minerales no cristalinos (sin orden de largo alcance) como alofán e imogolita controlan el almacenamiento y el movimiento del carbono orgánico del suelo (Yu y col., 2012). La estabilización de la materia orgánica se produce por medio de la adsorción sobre el área superficial específica, protegiéndola de la desintegración microbiana.

Además de la adsorción superficial, el entrampamiento de carbono orgánico en la estructura fractal del alofán haría menos accesible la materia orgánica para la degradación por la flora microbiana y el ataque de las enzimas (Chevallier y col., 2010); este fenómeno se debe a la estructura mesoporosa que se produce por el agregado de las partículas de alofán. La variación de carbono en los suelos alofánicos no es explicada por el contenido de limo y arcilla, más bien el alofán, el pH del suelo en agua y el contenido de aluminio explica la mayor variación de carbono del suelo, encontrándose una relación inversa entre el $\mathrm{pH}$ del suelo en agua y el $\mathrm{Al}$ (también Fe) complejados con materia orgánica del suelo (Garrido-Ramírez y col., 2012).

\subsection{Catálisis}

\subsubsection{Catálisis por reacciones tipo Fenton}

Las arcillas y el óxido de hierro son una alternativa a los catalizadores utilizados en reacciones (tipo 
Fenton) para la descontaminación de suelos, aguas, sedimento, así como efluentes industriales, debido a que son de bajo costo, abundantes en la naturaleza e inofensivo para ella. Los procesos de tipo Fenton implican la reacción de $\mathrm{Fe}^{2+}$ con peróxido de hidrógeno, originando la formación de radicales hidroxilos y $\mathrm{Fe}^{3+}$, el $\mathrm{Fe}^{3+}$ reacciona con peróxido formando $F e^{2+}$, el cual genera más radicales hidroxilos que son altamente oxidantes y capaces de descomponer un amplio espectro de compuestos orgánicos. Sobre esta base se ha estudiado la degradación de azul de metileno, usando alofán recubierto con óxidos de hierro; el alofán adsorbe el azul de metileno, mientras la interacción del Fe del alofán con el peróxido de hidrógeno da lugar a la formación de los radicales, los cuales descomponen el azul de metileno en moléculas orgánicas más pequeñas (Abidin y col., 2011).

El alofán recubierto con óxidos de hierro también se ha evaluado en la oxidación de atrazina (1Cloro-3-etil amino-5-isopropil amino-2,4,6-triazine) en un sistema electro-Fenton heterogéneo, usando electrodos de carbón vítreo y mostrando mayor eficiencia que un proceso Fenton heterogéneo. La atrazina es un herbicida orgánico que se encuentra como contaminante en las fuentes de agua subterránea y en los suministros de agua potable (GarridoRamírez y col., 2013). Garrido-Ramírez y col. (2012) y Garrido-Ramírez y col. (2016) han evaluado también la actividad catalítica de óxidos de hierro y óxidos de cobre soportados sobre alofán así como nanopartículas de $\mathrm{Fe}, \mathrm{Cu}$ y bimetálicas $\mathrm{Fe}-\mathrm{Cu}$, para la oxidación de fenol mediante reacciones heterogéneas electro-Fenton. En el primer caso se observó una influencia de la relación $\mathrm{Si} / \mathrm{Al}$ y su respectiva estructura, mientras que en el segundo caso se evidencia una mayor eficiencia cuando se utilizan nanopartículas bimetálicas en comparación a nanopartículas de $\mathrm{Cu}$ y Fe de forma independiente, esto debido a un efecto sinérgico.

\subsubsection{Fotocatálisis}

La fotocatálisis es una técnica de remediación que involucra la adsorción de radiación UV-visible, la cual permite la degradación de compuestos organoclorados. Se ha estudiado la degradación de tricloroetileno, un peligroso contaminante orgánico y acetaldehído en compósitos de alofán-titanio (Nishikiori y col., 2010; Nishikiori y col., 2015; Nishi- kiori y col., 2017). La presencia de alofán produce un incremento en la adsorción del titanio e inhibe la emisión de fosgeno $\left(\mathrm{COCl}_{2}\right)$ y dicloroacetil cloruro; productos intermedios de la descomposición de tricloroetileno, los cuales una vez adsorbidos en el alofán son degradados poco a poco luego de difundir hacia el titanio. La actividad fotocatalítica del compósito mejora con el tratamiento en medio ácido, lo cual se puede observar en la descomposición fotocatalítca de acetaldehído gaseoso (Ono y Katsumata, 2014).

Para la degradación fotocatalítica de acetaldehído gaseoso Hojamberdiev y col. (2014), utilizaron compósitos alofán-wakefieldite-(Ce), obtenidos por mezcla mecánica y por síntesis hidrotérmica, y observaron que ambos muestran una alta actividad fotocatalítica en comparación al alofán o wakefieldite $\left(\mathrm{CeVO}_{4}\right)-(\mathrm{Ce})$ por separado, esto debido a que el alofán en los compósitos produce un incremento de la adsorción. El compósito obtenido vía síntesis hidrotérmica muestra una mayor actividad fotocatalítica respecto al obtenido por mezcla mecánica, debido a una distribución más homogénea de wakefieldite-(Ce) y alofán.

En otro estudio para la fotodegradación de acetaldehído gaseoso Hojamberdiev y col. (2014) utilizan $\mathrm{Bi}_{2} \mathrm{WO}_{6}$-alofán y $\mathrm{BiOI}$-alofán también obtenidos por mezcla mecánica y por síntesis hidrotérmica. BiOI y BiOI-alofán descomponen el acetaldehído por completo dentro de 5 a 7 horas, mientras que $\mathrm{Bi}_{2} \mathrm{WO}_{6}$ y los compósitos obtenidos por mezcla y por síntesis descomponen el 75,5\%, 100\% y 85,6\% durante 8 horas, respectivamente. El alofán también contribuye en estos compósitos debido a la significativa adsorción de acetaldehído.

\subsubsection{Catálisis heterogénea}

La búsqueda de materiales energéticos como la biomasa es una opción a los combustibles fósiles, la hidrolisis de polisacáridos es un proceso para obtener monosacáridos, a partir de los cuales se puede obtener a su vez etanol. Okagi et al., (2011) estudiaron la hidrólisis de bambú, seda y cascarilla de arroz utilizando alofán sulfonado. En el bambú, la hemicelulosa fue descompuesta a xilosa, así como xilo-oligosacáridos; en el caso de la ceda los principales productos fueron xilosa y manosa, aunque también hubo producción de glucosa, galactosa y 
arabinosa. Mientras que, en la cascarilla del arroz, el producto principal fue arabinosa, aunque también se obtuvo xilosa y galactosa.

La degradación de compuestos como las siliconas o poli (dimetilsiloxano) en la presencia de arcillas, así como el efecto de la arcilla en la adsorción de los productos de degradación también ha sido estudiado. En un estudio en el que se utilizaron 12 diferentes arcillas, el alofán mostró un menor efecto en la actividad catalítica en comparación con caolinita, beidellita y nontronita, mientras que los productos de degradación fueron enlazados más fuerte a goethita y esmectita. El uso de alofán sintético promueve también la reducción de $\mathrm{K}_{2} \mathrm{PtCl}$ a platino $\left(P t^{0}\right)$ y actúa como un soporte de las nanopartículas de $P t^{0}$ de $2 \mathrm{~nm}$, obteniéndose de esta manera un compósito con potencial uso en catálisis heterogénea (Arakawa, Matsuura y Okamoto, 2014).

\subsection{Celdas fotocombustibles}

El uso de celdas fotocombustibles para generar electricidad por medio de la oxidación de materiales combustibles durante la irradiación UV es una tendencia en los últimos años. Los electrodos de las celdas fotocombustibles actúan como la fase que interactúa con el material combustible. En estos sistemas, la concentración del material combustible en la superficie del fotocatalizador utilizado es clave para mejorar la eficiencia de la conversión energética. Un método para incrementar la concentración es el uso de materiales adsorbentes. Aprovechando que el alofán tiene una gran área superficial, Nishikiori y col. (2012) y Nishikiori y col. (2014) estudiaron celdas combustibles utilizando electrodos elaborados a partir del compósito alofán-titanio y glucosa y almidón como materiales combustibles. En el primer caso el alofán adsorbe la glucosa transportándola luego hacia el titanio, en el cual su oxidación induce la electrogeneración, en el caso del uso de almidón también se mejora la generación de electricidad.

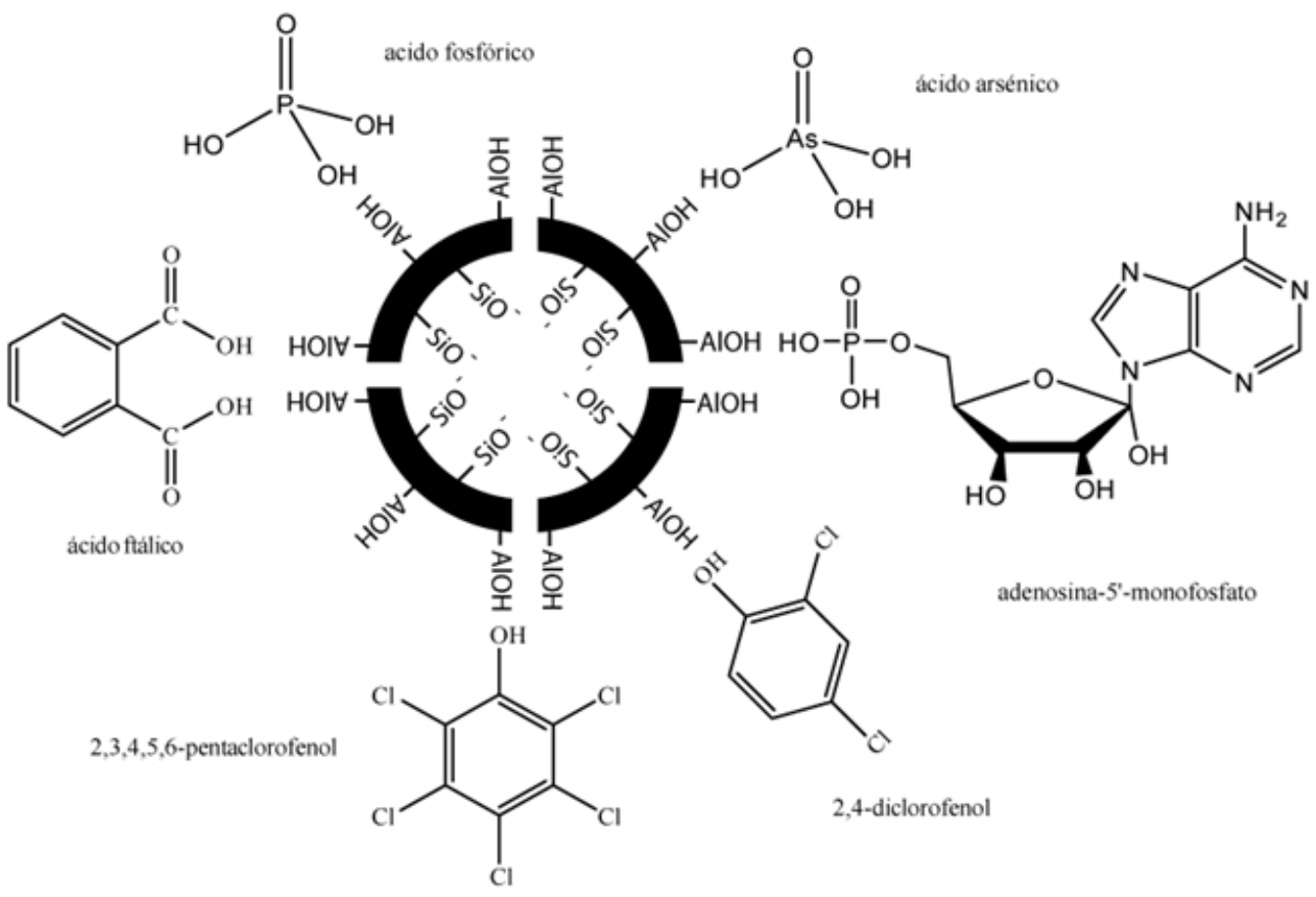

Figura 4. Diversas especies adsorbidas sobre alofán, la presencia de grupos $\mathrm{OH}$ en la mayoría de especies estudiadas permite el intercambio de ligandos y que las especies queden retenidas en la superficie del alofán. 


\subsection{Farmacología}

Las arcillas se han utilizado en la cosmética y productos industriales, pero en la actualidad su aplicación se está expandiendo de forma continua en la industria farmacéutica, la ingeniería de tejidos y el área médica. Al ser el alofán un material no tóxico y biocompatible es un buen candidato para aplicaciones médicas, ya que presenta propiedades bactericidas que pueden ser potenciadas por la formación de nanocompósitos basados en la inmobilización de nanopartículas de cobre y plata, también con propiedades bactericidas. En este contexto, el alofán ha sido utilizado para soportar agentes antibacterianos como nanopartículas de plata, observándose una fuerte actividad bactericida hacia Escherichia coli y Staphylococcus aureus (Cervini-Silva y col., 2015). Este efecto se debe a que la plata exhibe una fuerte actividad bactericida contra un amplio espectro de especies de hongos y bacterias, además de baja toxicidad, alta estabilidad térmica y baja volatilidad.

El alofán presenta propiedades antiinflamatorias al igual que la de otras arcillas como halloysita, debido a que su aplicación inhibe la formación de edema hasta entre un 39 a $60 \%$. Si bien el Fe presente en las estructura del alofán podría tener algún rol en este efecto no se han identificado aún los mecanismo, de tal manera que se pueda recomendar su uso con fines curativos (Cervini-Silva y col., 2015; Cervini-Silva y col., 2016). El alofán natural induce la peroxidación lipídica, la degradación oxidativa de los lípidos en las membranas celulares, y citotoxicidad de monocitos murinos, lo cual podría deberse a la presencia de $\mathrm{Fe}$ asociado a la superficie, y que podría generar especies reactivas de oxígeno (Cervini-Silva y col., 2014; Cervini-Silva y col., 2016; Toyota y col., 2017). Estos resultados han promovido estudios de la citotoxicidad de nanopartículas de alofán naturales y sintéticas contra células cancerosas humanas, con la intención de utilizar las nanopartículas de alofán como un nanoportador para la administración de fármacos.

El estudio de hidrogeles basados en moléculas de ADN y alofán natural ha sido abordado por Kawachi y col. (2013), en donde las moléculas de ADN se adsorben en la superficie, enrollándose alrededor de las partículas de alofán y formando el hidrogel. La adsorción es facilitada por la interacción entre los grupos fosfato del ADN y los grupos presentes en las perforaciones de la pared del alofán. El estudio de este tipo de hidrogeles podría ser útil para la generación de nuevas formas de liberación de fármacos con dosis específicas.

\subsection{Inmovilización de enzimas}

Por su gran área superficial el alofán es un material que sirve como un soporte útil de múltiples tipos de especies, entre estas las enzimas. De esta manera, el alofán se ha evaluado como material de soporte para inmovilizar fosfatasa ácida y evaluar la mineralización de fósforo orgánico de estiércol de ganado descompuesto con complejos de arcillas y nanoarcilla-fosfatasa ácida. Se ha observado que la inmovilización incrementa tanto la actividad enzimática específica como la cinética de la mineralización de fósforo orgánico (Calabi-Floody y col., 2012). Se han obtenido complejos sintéticos por la interacción entre fosfatasa ácida, ácido tánico y alofán natural con el fin de tener un mejor entendimiento de la mineralización de fósforo orgánico. La inmovilización de la fosfatasa en ácido tánico disminuye la actividad enzimática y afecta la cinética, mientras que la inmovilización en alofán incrementa la actividad de la enzima en comparación con la enzima libre, lo que indicaría que este tiene un efecto protector en la conformación de la enzima. La presencia de Mn y Mo en la actividad catalítica de la fosfatasa ácida inmovilizada, en el caso del Mn, disminuye la velocidad en comparación con la fosfatasa libre cuando se añade al mismo tiempo que la enzima; sin embargo, cuando se añade después de la interacción no se observan efectos, no así para el caso de Mo, aunque el efecto es menor cuando se añade después de la interacción enzima arcilla (Rosas y col., 2008).

Se ha estudiado también la estabilización de la actividad de dos fitasas microbianas comerciales luego de su inmovilización en alofán sintético, alofán cubierto con óxidos de hierro y montmorillonita natural. La inmovilización mejora la estabilidad térmica y la resistencia a la proteólisis, además la actividad residual de ambas fitasas fue más alta bajo condiciones ácidas (Menezes-Blackburn y col., 2011).

Los nanomateriales se han convertido en materiales omnipresentes, pues en la actualidad existen productos comerciales que contienen algún tipo 
de nanopartícula (Heiligtag y Niederberger, 2013; Nguyen y S., 2020). En Ecuador se consumen productos que contienen nanomateriales, y como en la mayoría de ocasiones no se generan productos de este tipo, a pesar de contar con instituciones de educación superior con capacidad para desarrollar investigación en esta área y con una fuente que puede proveer de estos materiales.

Existe un creciente interés por el desarrollo de nanoproductos naturales, por ejemplo, en los campos de la fito y la nanotecnología, medicina, nutrición, cosmética y agricultura (Griffin y col., 2018). En este contexto, el alofán puede ser utilizado como un material de refuerzo para el diseño de empaques degradables, en agricultura para la liberación controlada de fertilizantes, en medicina para la liberación de fármacos, en el área petrolera como nanofluido para la perforación de pozos, como nanocatalizadores en la refinación de crudo (Rashidi y col., 2018) y en el área ambiental para la reducción de la contaminación (Wilson, 2018).

Es necesario la creación de grupos multidisciplinarios, líneas, programas y proyectos de investigación en el área de nanomateriales, así como el apoyo de los organismos correspondientes que apoyen estudios de este tipo de suelos y de los yacimientos que tienen una considerable concentración de nanopartículas naturales.

\section{Conclusiones}

Las investigaciones nacionales realizadas en torno a suelos de origen volcánico ecuatorianos se han enfocado en temas que no tienen relación con la nanopartícula de alofán, apenas en los últimos años se evidencian investigaciones sobre alofán. Sin embargo, las investigaciones son realizadas por instituciones ubicadas fuera del país.

El alofán cuenta con propiedades únicas y versátiles como gran área superficial, carga variable, alta retención de humedad, alta porosidad. Se puede extraer a partir de fuentes naturales, pero también se pueden obtener por los diferentes métodos de síntesis mencionados. Tiene potenciales de aplicación para remediación ambiental, extracción de petróleo, catálisis, fotocatálisis, electrocatálisis, empaques inteligentes, nanosensores, soporte de enzimas, fár- macos y fertilizantes.

\section{Agradecimientos}

A la Facultad de Ciencias de la Escuela Superior Politécnica de Chimborazo.

Al Instituto de Investigaciones de la Escuela Superior Politécnica de Chimborazo.

A la Secretaría de Educación Superior, Ciencia, Tecnología e Innovación (SENESCYT) del Ecuador.

\section{Referencias}

Abidin, Z. y col. (2011). «B21 Fenton-like Reaction on Degradation of Organic Dye by Natural Allophane». En: Abstracts of annual meeting of The Clay Science Society of Japan 55th Annual Meeting of The Clay Science Society of Japan, 124-125. Online:https://bit.ly/3qflN84.

Alvarado, A., R. Mata y M. Chinchilla (2014). «Arcillas identificadas en suelos de Costa Rica a nivel generalizado durante el período 1931-2014: I. Historia, metodología de análisis y mineralogía de arcillas en suelos derivados de cenizas volcánicas». En: Agronomía Costarricense 38.1, 75-106. Online:https:/ / bit.ly/3liEdkU.

Arakawa, S., Y. Matsuura y M. Okamoto (2014). «Allophane-Pt nanocomposite: Synthesis and MO simulation». En: Applied clay science 95, 191-196.Online:https:/ / bit.ly/3fRbrXk.

Araujo-Bilmonte, Elking, Liceth Huertas-Tulcanaza y Kenny Párraga-Stead (2020). «Análisis de la producción científica del Ecuador a través de la plataforma Web of Science». En: Cátedra 3.2, 150-165. Online:https://bit.ly/2JwxkiJ.

Baldermann, A. y col. (2018). «Removal of barium, cobalt, strontium, and zinc from solution by natural and synthetic allophane adsorbents». En: Geosciences 8.9, 309. Online:https : / / bit . ly / 2JpRDyn.

Buytaert, W., J. Deckers y G. Wyseure (2007). «Regional variability of volcanic ash soils in south Ecuador: The relation with parent material, climate and land use». En: Catena 70.2, 143-154. Online:https://bit.ly/3nf75fD. 
Calabi-Floody, M. y col. (2012). «Improving bioavailability of phosphorous from cattle dung by using phosphatase immobilized on natural clay and nanoclay». En: Chemosphere 89.6, 648-655. Online:https:/ / bit.ly/348uSpR.

Calvache, M (2014). «El suelo y la productividad agrícola en la sierra del Ecuador». En: XIV Congreso Ecuatoriano de la Ciencia del Suelo.

Calvache, M. (2015). «Manejo sostenible de los suelos del Ecuador». En: VII Congreso Sudamericano de Agronomía, Guayaquil.

Cervini-Silva, J. y col. (2014). «Lipid peroxidation and cytotoxicity induced by respirable volcanic ash». En: Journal of hazardous materials 274, 237-246. Online:https:/ / bit.ly/347GQQI.

Cervini-Silva, J. y col. (2015). «The antiinflammatory activity of natural allophane». En: Applied Clay Science 105, 48-51. Online:https: //bit.ly/37cQfIE.

Cervini-Silva, J. y col. (2016). «Anti-inflammatory, antibacterial, and cytotoxic activity by natural matrices of nano-iron (hydr) oxide/halloysite». En: Applied Clay Science 120, 101-110. Online:https://bit.ly/3ne763x.

Chevallier, Tiphaine y col. (2010). «Organic carbon stabilization in the fractal pore structure of Andosols». En: Geoderma 159.1-2, 182-188. Online:https://bit.ly/3nqJbO4.

Elhadi E and, Matsue N. y T. Henmi (2000). «Adsorption of molybdate on nano-ball allophane». En: Clay science 11.2, 189-204. Online:https:/ / bit.ly / 2W9P46u.

FAO (2014). Atlas de suelos de América Latina y el Caribe. Oficina de Publicaciones de la Unión Europea.

- (2015). Base referencial mundial del recurso suelo 2014 Sistema internacional de clasificación de suelos para la nomenclatura de suelos y la creación de leyendas de mapas de suelos.

Garrido-Ramírez, E. y col. (2012). «Catalytic wet peroxide oxidation of phenol over iron or copper oxide-supported allophane clay materials: Influence of catalyst $\mathrm{SiO} 2 / \mathrm{Al} 2 \mathrm{O} 3$ ratio». En: $\mathrm{Mi}$ croporous and mesoporous materials 162, 189-198. Online:https:/ / bit.ly/3nj88Lm.

Garrido-Ramírez, E. y col. (2013). «Characterization of nanostructured allophane clays and their use as support of iron species in a heterogeneous electro-Fenton system». En: Applied clay science 86, 153-161. Online:https://bit.ly/37eDhKn.
Garrido-Ramírez, E. y col. (2016). «Preparation and characterization of bimetallic $\mathrm{Fe}-\mathrm{Cu}$ allophane nanoclays and their activity in the phenol oxidation by heterogeneous electro-Fenton reaction». En: Microporous and Mesoporous Materials 225, 303-311. Online:https://bit.ly/3mmd5C9.

Gonzáles, A. (2015). «Los suelos del Ecuador.» En: XX Congreso Latinoamericano y XVI Congreso Peruano de La Ciencia Del Suelo; Cusco.

González, A. (2010). «Suelos de Ecuador». En: 1er Taller Latinoamericano Globalsoilmap.Net, Rio de Janeiro.

Griffin, Sharoon y col. (2018). «Natural nanoparticles: A particular matter inspired by nature». En: Antioxidants 7.1, 3. Online:.

Gutiérrez Coronado, J. (2018). "El mundo "Nano" de Ecuador ¿Cómo de grande es?» En: Momento 56E, 65-80. Online:https://bit.ly/2LBlG7j.

Hashizume, H. y B. Theng (2007). «Adenine, adenosine, ribose and 5-AMP adsorption to allophane». En: Clays and Clay Minerals 55.6, 599-605. Online:https:/ / bit.ly/383gGjh.

Hashizume, H., B. Theng y A. Yamagishi (2002). "Adsorption and discrimination of alanine and alanyl-alanine enantiomers by allophane». En: Clay Minerals 37.3, 551-557. Online:https:/ / bit. ly/3ns1EK8.

Heiligtag, F. y M. Niederberger (2013). «The fascinating world of nanoparticle research». En: $M a-$ terials Today 16.7-8, 262-271. Online:https: / / bit. ly/37X3vjT.

Henmi, T. y K. Wada (1976). «Morphology and composition of allophane». En: American Mineralogist 61.5-6, 379-390. Online:https:/ / bit.ly/383B3wu.

Hojamberdiev, M. y col. (2014). «Preparation of Bi2WO6-and BiOI-allophane composites for efficient photodegradation of gaseous acetaldehyde under visible light». En: Applied clay science 101, 38-43. Online:https://bit.ly/37ZIa9p.

Huang Y.and Lowe, D.and Churchman G.and Schipper L. y A. Rawlence N.and Cooper (2014). «Carbon storage and DNA adsorption in allophanic soils and paleosols». En: Soil Carbon. Springer, 163-172. Online:https : / / bit . ly / 3a3BtG1.

Iyoda, F. y col. (2012). "Synthesis and adsorption characteristics of hollow spherical allophane nano-particles». En: Applied Clay Science 56, 77-83. Online:https://bit.ly/3qMQeTD.

Jansen, B. y K. Nierop (2009). «Methyl ketones in high altitude Ecuadorian Andosols confirm ex- 
cellent conservation of plant-specific n-alkane patterns». En: Organic Geochemistry 40.1, 61-69. Online:https:/ / bit.ly/37e9Fgg.

Kaufhold, S. y col. (2010). "Allophane compared with other sorbent minerals for the removal of fluoride from water with particular focus on a mineable Ecuadorian allophane». En: Applied clay science 50.1, 25-33. Online:https: / / bit.ly / 3nsNUPH.

Kawachi, T. y col. (2013). «Preparation and characterization of DNA/allophane composite hydrogels». En: Colloids and Surfaces B: Biointerfaces 112, 429-434. Online:https:/ / bit.ly/3gIH4mh.

Matsuura, Y., S. Arakawa y M. Okamoto (2014). «Single-stranded DNA adsorption characteristics by hollow spherule allophane nanoparticles: $\mathrm{pH}$ dependence and computer simulation». En: Applied clay science 101, 591-597. Online:https://bit.ly/3qQaxPT.

Matsuura, Y. y col. (2013). «DNA adsorption characteristics of hollow spherule allophane nanoparticles». En: Materials Science and Engineering: C 33.8, 5079-5083. Online:https : / / bit . ly / 3ngIIOG.

Menezes-Blackburn, D. y col. (2011). "Activity stabilization of Aspergillus niger and Escherichia coli phytases immobilized on allophanic synthetic compounds and montmorillonite nanoclays». En: Bioresource technology 102.20, 9360-9367. Online:https://bit.ly/2JYqkLS.

Nguyen, T. y Rajendran. S. (2020). «Chapter 23 - Current commercial nanocosmetic products». En: Nanocosmetics. Micro and Nano Technologies. Elsevier.

Nishikiori, H. y col. (2010). «Removal of detergents and fats from waste water using allophane». En: Applied clay science 47.3-4, 325-329. Online:https: //bit.ly/3a9ad9c.

Nishikiori, H. y col. (2012). «Photofuel Cells Using Allophane-Titania Nanocomposites». En: Chemistry Letters 41.7, 725-727. Online:https: / / bit. ly/2K3v17l.

Nishikiori, H. y col. (2014). «Reaction in photofuel cells using allophane-titania nanocomposite electrodes». En: Applied Catalysis B: Environmental 147, 246-250. Online:https:/ / bit.ly/37e4nkK.

Nishikiori, H. y col. (2015). «Degradation of Trichloroethylene Using Allophane-Titania Nanocomposite Supported on Porous Filter». En: Chemistry Letters 44.5, 639-641. Online:https: / / bit. ly/3meDx0c.
Nishikiori, H. y col. (2017). «Influence of allophane distribution on photocatalytic activity of allophane-titania composite films». En: Applied Clay Science 146, 43-49. Online:https: / / bit.ly / 2Wb3EKV.

Ono, Y. y K. Katsumata (2014). «Enhanced photocatalytic activity of titanium dioxide/allophane mixed powder by acid treatment». En: Applied clay science 90, 61-66. Online:.

Opiso, E., T. Sato y T. Yoneda (2009). «Adsorption and co-precipitation behavior of arsenate, chromate, selenate and boric acid with synthetic allophane-like materials». En: Journal of hazardous materials 170.1, 79-86. Online:https: / / bit. ly/3qRuOVp.

Pérez, N. y col. (2016). «Identification of allophane and other semi-crystalline and amorphous phases on pre-Hispanic Mexican adobe earth bricks from Cholula, Mexico». En: Microchemical Journal 126, 349-358. Online:https: / / bit.ly / 2WcA1c8.

Podwojewski, P. y N. Germain (2005). «Short-term effects of management on the soil structure in a deep tilled hardened volcanic-ash soil (cangahua) in Ecuador». En: European journal of soil science 56.1, 39-51. Online:https : / / bit . ly / 3oP9A8E.

Podwojewski, P. y col. (2002). «Overgrazing effects on vegetation cover and properties of volcanic ash soil in the páramo of Llangahua and La Esperanza (Tungurahua, Ecuador)». En: Soil Use and Management 18.1, 45-55. Online:https:/ / bit. ly/3gGOWF5.

Poncelet, O. y J. Jouhannaud (2013). Use of nanoparticles for the long-term "dry "storage of peroxide radicals (Patent No. US 20130142996 A1). URL: https: / / bit.ly/3qRIH5S.

Rashidi, A. y col. (2018). Nanotechnology in Oil and Gas Industries.

Reinert, L. y col. (2011). "Characterization and boron adsorption of hydrothermally synthesised allophanes». En: Applied clay science 54.3-4, 274-280. Online:https://bit.ly/3oP7Ps9.

Rosas, A. y col. (2008). «Catalytic behaviour of acid phosphatase immobilized on natural supports in the presence of manganese or molybdenum». En: Geoderma 145.1-2, 77-83. Online:https: / / bit. ly/3oOMJKm.

Saeki, K., M. Sakai y S. Wada (2010). «DNA adsorption on synthetic and natural allophanes». En: 
Applied Clay Science 50.4, 493-497. Online:https: //bit.ly/2WcI3li.

Shukla, E. y col. (2013). «A comparative study of arsenate and phosphate adsorption on nano-ball allophane». En: Clay Science 17.4, 83-91. Online:https://bit.ly/3gMy6Vo.

Silva-Yumi, J. y col. (2018). «Kinetics, adsorption and desorption of $\mathrm{Cd}$ (II) and $\mathrm{Cu}$ (II) on natural allophane: effect of iron oxide coating». En: Geoderma 319, 70-79. Online:https : / / bit . ly / 3qVLtHa.

Toyota, Y. y col. (2017). "Cytotoxicity of natural allophane nanoparticles on human lung cancer A549 cells». En: Applied Clay Science 135, 485-492. Online:https://bit.ly/386ovVw.

Triomphe, B. y L. Livermore (2005). «Mineralogical control of organic carbon dynamics in a volcanic ash soil on La Rèunion». En: European Journal of Soil Science 56, 689-703. Online: https: / / bit.ly / 384P512.

Vaca, J. y S. Lalangui (2018). «Evaluación de métodos de activación del alofán de Santo Domingo de los Tsáchilas». Trabajo de titulación previo a la obtención del íítulo de Ingeniero Químico. Universidad Central del Ecuador.

Vistoso, E. y col. (2012). "Competitive sorption of molybdate and phosphate in Andisols». En:
Journal of soil science and plant nutrition 12.1, 59-72. Online:https:/ / bit.ly /2Lu4FeZ.

Wilson, N. (2018). «Nanoparticles: environmental problems or problem solvers?» En: Bioscience 68.4, 241-246. Online:https:/ / bit.ly/3a46bPn.

Yu-Huang, H. y col. (2016). «DNA adsorption by nanocrystalline allophane spherules and nanoaggregates, and implications for carbon sequestration in Andisols». En: Applied Clay Science 120, 40-50. Online:https:/ / bit.ly/3gKcN6E.

$\mathrm{Yu}, \mathrm{G}$. y col. (2012). «Binding of organic ligands with Al (III) in dissolved organic matter from soil: implications for soil organic carbon storage». En: Environmental science \& technology 46.11, 6102-6109. Online:https://bit.ly/3me5LIv.

Yuan, G. y S. Wada (2012). "Allophane and imogolite nanoparticles in soil and their environmental applications». En: Nature's nanostructures. Pan Stanford, Singapore, 494-515. Online:https: / / bit. ly/3qWDD0i.

Zaenal, A., N. Matsue y T. Henmi (2013). «Adsorption of amines on nano-ball allophane and its molecular orbital analysis». En: Clay Science 17.3, 67-73. Online:https://bit.ly/3r3xv6q.

Zehetner, F., W. Miller y L. West (2003). «Pedogenesis of volcanic ash soils in Andean Ecuador». En: Soil Science Society of America Journal 67.6, 1797-1809. Online:https://bit.ly/3abT8v9. 